\title{
Quantum dot formation on a strain-patterned epitaxial thin film
}

\author{
S. M. Wise, J. S. Lowengrub, and J. S. Kim \\ Mathematics Department, University of California, Irvine, California 92697-3875 \\ K. Thornton \\ Department of Materials Science and Engineering, University of Michigan, Ann Arbor, \\ Michigan 48109-2136 \\ P. W. Voorhees \\ Materials Science and Engineering Department, Northwestern University, Evanston, Illinois 60208-3108
}

W. C. Johnson

Department of Materials Science and Engineering, University of Virginia, Charlottesvile, Virginia 22904-4745

(Received 18 July 2005; accepted 22 August 2005; published online 19 September 2005)

\begin{abstract}
We model the effect of substrate strain patterning on the self-assembly of quantum dots (QDs). When the surface energy is isotropic, we demonstrate that strain patterning via embedded substrate inclusions may result in ordered, self-organized QD arrays. However, for systems with strong cubic surface energy anisotropy, the same patterning does not readily lead to an ordered array of pyramids at long times. We conclude that the form of the surface energy anisotropy strongly influences the manner in which QDs self-assemble into regular arrays. (C) 2005 American Institute of Physics.
\end{abstract}

[DOI: $10.1063 / 1.2061852$ ]

When a single-component thin film is coherently bonded to a planar lattice-mismatched substrate, small perturbations with certain wavelengths on the surface of the film will grow. The process is well-understood by the linear theory developed by Asaro, Tiller, Grinfeld (ATG). ${ }^{1,2}$ The ATG theory must be modified when the film's surface comes near the substrate because the substrate interacts energetically with the trough of the surface wave. If the film wets the substrate and the film is initially not too thick, islands can develop without forming dislocations and subsequently coarsen as mass flows along the wetting layer. This scenario is generally termed Stranski-Krastanow (SK) growth. ${ }^{3}$ In SK growth the film must exceed a critical thickness before it becomes unstable because of the wetting layer effect, which tends to hold the film flat against the substrate. The kinetics of SK island growth depend on a number of factors, including the initial thickness of the film; the relative sizes of the rate of attachment of adatoms during vapor deposition and the rate associated with surface diffusion; the competition between the strain and surface energies, and their associated anisotropies; and the wetting layer thickness.

The interest in SK growth lies with its recognized importance as a potential pathway for the formation of ordered quantum dot (QD) arrays. Recent experimental work has suggested that the spatial ordering of islands may be controlled by patterning the substrate through strain localization. ${ }^{4-6}$ In this work we investigate the controllability of forming ordered island arrays using strain patterning by embedded inclusions in the substrate, which may be formed by a multilayer deposition process. ${ }^{6}$ The effect of strain patterning in two dimensions on ordered array development was investigated by Wise et $a l^{7}$ The use of a buried pyramidal island to produce a single QD was investigated using a 2 +1 dimensional model in Quek and Liu. ${ }^{8}$

We use a three-dimensional Cahn-Hilliard-type equation with a source term modeling mass deposition. In order to simulate strong surface energy anisotropy, a high-order cor- ner energy is added to regularize the instability that can arise from missing orientations on the Wulff shape, yielding a sixth-order evolution equation. The three-dimensional cubicelastic equilibrium equations are coupled to the evolution and describe the state of strain arising due to epitaxial misfit and the buried inclusions. Wetting is described through an explicit wetting potential, such that the bulk free energy density has a double-welled form outside the substrate and a single-welled form inside the substrate. The phase-fields $\phi$ and $c$ describe the system: substrate, $\phi=1, c=1$; film, $\phi=0$, $c=1$; and vapor, $\phi=0, c=0$. The nondimensional governing equations are,

$$
\begin{aligned}
& c_{, t}=\frac{1}{\epsilon_{0}^{2}} \nabla \cdot(M(\phi, c) \nabla \mu)+n_{3} \frac{V}{\epsilon_{0}} c^{2}(1-c)^{2}, \\
& \begin{aligned}
& M(\phi, c)=16 c^{2}(1-c)^{2}(1-\phi), \\
& \mu= f_{, c}(\phi, c)+Z \epsilon_{0} W_{, c}(\phi, c, E)-\epsilon_{0}^{2} \nabla^{2} c \\
&-\nabla \cdot(\mathcal{A}(\phi, \mathbf{n}) \nabla c)+\chi_{0}^{2} \nabla^{4} c, \\
& f(\phi, c)=(1-\phi) c^{2}(1-c)^{2} / 4+\phi \omega_{s}(c-1)^{2} / 4,
\end{aligned}
\end{aligned}
$$

where $\epsilon_{0}$ is the gradient energy parameter; $M$ is the surface mobility; $n_{3}$ is the component of the surface unit normal in the direction of mass deposition; $V$ is the random deposition rate; $\mu$ is the chemical potential; $f$ is the bulk free energy density; $Z$ measures the relative strength of the elastic energy to the surface energy on a characteristic length scale; $W$ is the elastic energy density; $\mathcal{A}$ is the surface anisotropy matrix; $\mathbf{n}=\left(n_{1}, n_{2}, n_{3}\right)^{T}=-\nabla c /\|\nabla c\| ; \chi_{0}$ is a corner regularization parameter; and $\omega_{s}$ is the wetting energy parameter. Previously published continuum-level models have either been two s, $^{7,10}$ or 2+1-dimensional. ${ }^{8,11-16}$ Ours is a fully three-dimensional phase-field model of QD formation in an epitaxial thin film capable of handling strong interfacial anisotropy in a consistent manner. Very recently Ratz et al. ${ }^{17}$ have used a three- 


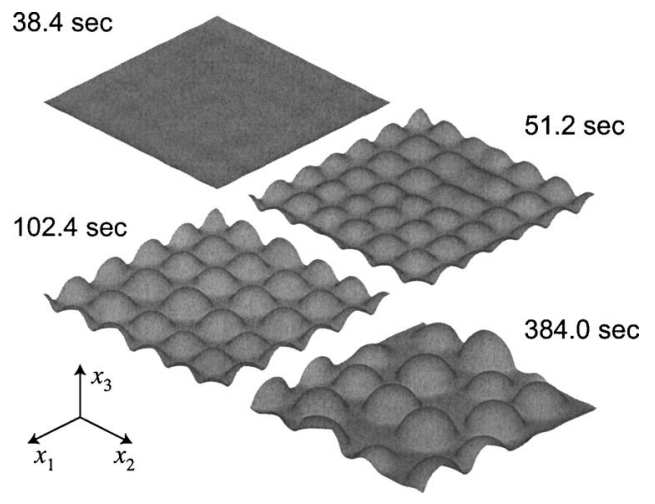

FIG. 1. Snap shots of an evolving isotropic film on a flat, unpatterned substrate.

dimensional viscous Cahn-Hilliard equation to model QD formation, but they do not consider strong surface anisotropy or substrate patterning.

We solve this system of equations using an efficient nonlinear multigrid method ${ }^{7,18}$ on a locally refined Cartesian mesh. In all of the following simulations we use periodic boundary conditions in the plane of the substrate. We use a prescribed substrate thickness, with clamped elastic boundary conditions at the bottom, of sufficient size such that increasing the thickness does not noticeably change the evolution of the film. The elastic boundary conditions at the top of the computational domain are traction-free. For the order parameter $c$ and chemical potential $\mu$ homogeneous Neumann boundary conditions are used at the top and bottom of the domain.

The physical parameters are chosen for the $\mathrm{SiGe} / \mathrm{Si}$ system, ${ }^{19}$ with the exception of those describing the surface energy. For simplicity, we assume the surface energy is cubic anisotropic. The periodic domain is $320 \mathrm{~nm}$ in length and width, and the total process time is about $384 \mathrm{~s}$. The vapor phase is modeled as a very soft elastic solid, with the ratio of the elastic stiffnesses between the film/substrate and the vapor being $10^{4}$. The buried inclusions are assumed coherent, with the same misfit as the film. The diffuse interface thickness is approximately $10 \mathrm{~nm}$ based on the choice of $\epsilon_{0}$. In the locally refined region there are at least five points across the interface, which give adequate resolution based on our refinement studies. To achieve smaller interface thicknesses, more efficient numerical solvers are currently being developed that incorporate adaptive mesh refinement and parallelization. In all simulations the substrate is a flat (001) plane and the initial film surface is parallel to the substrate with thickness equal to $12.5 \mathrm{~nm}$, which is above the critical wetting thickness. All figures show the $c=0.5$ isosurface.

In Fig. 1, a film is shown evolving on an unpatterned substrate. At early times the film undergoes the ATG instability and a relatively ordered arrangement of QDs emerges. The dots are initially aligned in the elastically soft $\langle 100\rangle$ directions. At later times the dots coarsen yielding irregular island distributions. The disordered arrangement of the dots is a result of the combination of the random nature of the deposition and coarsening processes. Thus from this simulation, the elastic anisotropy is not sufficient to guarantee an ordered arrangement of QDs at long times.

For the simulation of Fig. 2, the substrate is strainpatterned using a spherical inclusion of radius $30 \mathrm{~nm}$ buried $50 \mathrm{~nm}$ beneath the substrate surface. The ATG instability is

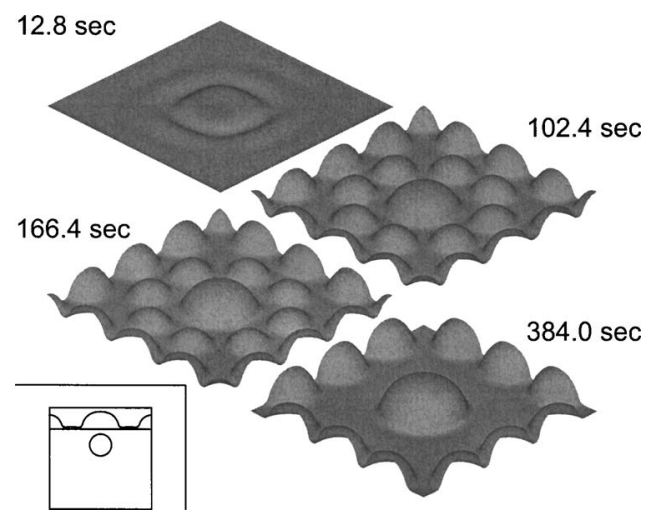

FIG. 2. Snap shots of an evolving isotropic film on a substrate strainpatterned using a single buried inclusion. Inset, $x_{1}$ slice through the middle of the computational domain.

enhanced and localized by the strain-patterning, and surface perturbations appear much earlier compared to the case with no patterning (Fig. 1). A regular pattern emerges. However, in contrast to the unpatterned case, as the system coarsens the arrangement maintains more regularity. The strain localization results in a dominant QD above the inclusion, surrounded by ordered satellites that form on the edges of the domain, aligned in the elastically soft directions. Since the configuration is periodic, this corresponds to an arrangement of a doubly periodic array of QDs. Although it seems likely that the satellite dots will coarsen at later times, the time scale for coarsening appears to be significantly longer than the simulated time. The strain patterning also makes the system less susceptible to the randomness continually introduced by the deposition.

The evolution with two embedded inclusions is shown in Fig. 3. The spheres have the same radius and depth as in Fig. 2 , but are placed along a diagonal of the periodic domain. Now the patterning results in the formation of two larger QDs above the inclusions. As the system coarsens two satellite dots emerge, resulting in a doubly periodic four-dot configuration. Thus from two inclusions, a four-dot pattern may be produced, leading to an array of dots with a length scale smaller than the initial strain patterning.

In Fig. 4 the effect of surface energy anisotropy is shown. The cubic anisotropic coefficient is taken to be large enough so that there are missing orientations on the Wulff shape. There is an embedded inclusion, with the same geometry as in Fig. 2. The flat initial film is unstable due both to an ATG-type instability and to surface energy anisotropy.

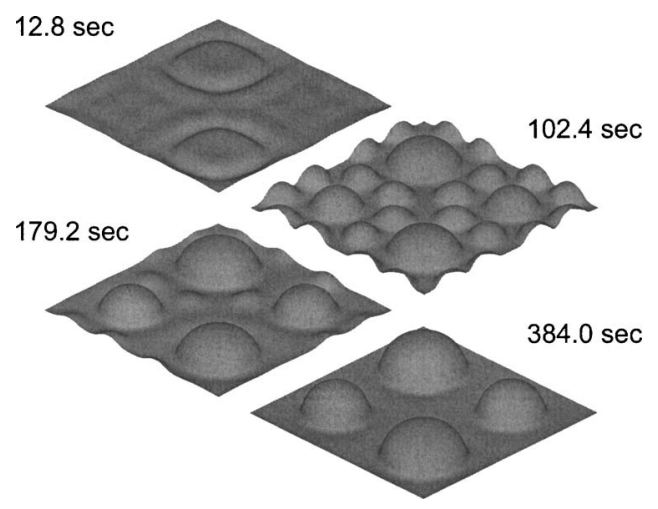

FIG. 3. An evolving isotropic film on a substrate strain-patterned using two coherent inclusions. 


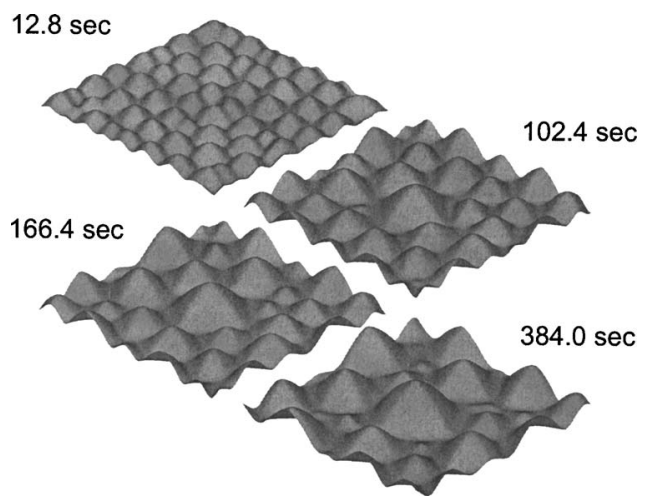

FIG. 4. An evolving film, with strongly anisotropic surface energy, on a patterned substrate with one buried inclusion.

The latter is because the film's initial orientation has a large energy and is missing from the Wulff shape. The surface perturbations at early times are less localized than in the isotropic case. The evolution is influenced by the random deposition flux to a greater degree than the isotropic case because the strong surface energy anisotropy allows the growth of perturbations with small wavelengths that would normally be damped in the ATG instability for the isotropic case. Localization is only seen at later times, as a large pyramidal QD forms above the inclusion. Unlike the case with isotropic surface energy, this strain-patterned system does not seem to result in an ordered arrangement of pyramids at long times. In addition to the enhanced instability due to strong surface energy anisotropy, the elastically soft directions correspond to high energy orientations on the islands, which may further contribute to the lack ordering. Thus, the form of the surface energy anisotropy strongly influences the manner in which QDs self-assemble into regular arrays. This result is similar to that obtained earlier for deposition on morphologically patterned substrates. ${ }^{9}$ Thus, it is clear that the magnitude of the surface energy anisotropy plays an important role in the efficacy of many methods for directed self assembly. Further, our results suggest that, for this strain patterning geometry, an ordering transition occurs at a critical value of the anisotropy coefficient (which depends on $\chi_{0}$ and $\left.\epsilon_{0}\right)$ and will be the subject of future investigation.

We have shown that embedded strain centers can be used to direct the self-assembly of quantum dots on surfaces. In particular, these strain centers can be used to produce a pattern that is of smaller scale than that of the strain centers, thus avoiding the challenge of patterning at the size scale of the dots. However, the directed self-assembly of dots can be more difficult in systems with strongly anisotropic surface energy.

We gratefully acknowledge the support of the National Science Foundation through the Center for the Design of Nanoscopic Materials, Grant DMR0080016, and the Divisions of Materials Research and Mathematical Sciences.

${ }^{1}$ R. Asaro and W. Tiller, Metall. Trans. 3, 1789 (1972).

${ }^{2}$ M. Grinfeld, Dokl. Akad. Nauk SSSR 290, 1358 (1982).

${ }^{3}$ D. Eaglesham and M. Cerullo, Phys. Rev. Lett. 64, 1943 (1990).

${ }^{4}$ M. DeSeta, G. Capellini, and F. Evangelisti, Phys. Rev. B 71, 115308 (2005).

${ }^{5}$ J. Tersoff, C. Teichert, and M. Lagally, Phys. Rev. Lett. 76, 1675 (1996).

${ }^{6}$ S. Kiravittaya, H. Heidemeyer, and O. Schmidt, Appl. Phys. Lett. 86, 263113 (2005).

${ }^{7}$ S. Wise, J. Lowengrub, J. Kim, and W. Johnson, Superlattices Microstruct. 36, 293 (2004).

${ }^{8}$ S. Quek and G. Liu, Thin Solid Films 497, 297 (2005).

${ }^{9}$ J. Eggleston, Ph.D. thesis, Northwestern University (2001).

${ }^{10}$ J. Eggleston and P. W. Voorhees, Appl. Phys. Lett. 80, 306 (2002).

${ }^{11}$ Y. Zhang, Phys. Rev. B 61, 10388 (2000).

${ }^{12}$ P. Liu, Y. Zhang, and C. Lu, Appl. Phys. Lett. 80, 3910 (2002).

${ }^{13}$ P. Liu, Y. Zhang, and C. Lu, Phys. Rev. B 67, 165414 (2003).

${ }^{14}$ P. Liu, Y. Zhang, and C. Lu, Surf. Sci. 526, 375 (2003).

${ }^{15}$ A. Golovin, M. Levine, T. Savina, and S. Davis, Phys. Rev. B 70, 235342 (2004).

${ }^{16}$ A. Ramasubramaniam and V. Shenoy, J. Appl. Phys. 95, 7813 (2004).

${ }^{17}$ A. Rätz, A. Ribalta, and A. Voigt, J. Comput. Phys. (in review).

${ }^{18}$ J. Kim, K. Kang, and J. Lowengrub, J. Comp. Physiol. 193, 511 (2003).

${ }^{19}$ B. Spencer, P. Voorhees, and J. Tersoff, Phys. Rev. B 64, 235318 (2001). 